\title{
COMPARISON OF ANALYTICAL SOLUTIONS FOR CMSMPR CRYSTALLIZER WITH QMOM POPULATION BALANCE MODELING IN FLUENT
}

\author{
Bin Wan ${ }^{1}$, Terry A. Ring ${ }^{1, *}$, Kumar M. Dhanasekharan ${ }^{2}$ and Jayanta Sanyal ${ }^{2}$ \\ ${ }^{1}$ Department of Chemical Engineering, University of Utah, Salt Lake City, UT 84112 \\ ${ }^{2}$ Fluent Inc., 10 Cavendish Court, Lebanon, NH 03766 \\ *Author to whom correspondence should be addressed. E-mail: T.Ring@m.cc.utah.edu
}

\begin{abstract}
Fluent version 6.2 computational fluid dynamics environment has been enhanced with a population balance capability that operates in conjunction with its multiphase calculations to predict the particle size distribution within the flow field. The population balance is solved by the quadrature method of moments (QMOM). Fluent's prediction capabilities are tested by using a 2-dimensional analogy of a constantly stirred tank reactor with a fluid flow compartment that mixes the fluid quickly and efficiently using wall movement and has a feed stream and a product stream. The results of these Fluent simulations using QMOM population balance solver are compared to steady state analytical solutions for the population balance in a stirred tank where 1) growth, 2) aggregation, and 3) breakage, take place separately and 4) combined nucleation and growth and 5) combined nucleation, growth and aggregation take place. The results of these comparisons show that the moments of the population balance are accurately predicted for nucleation, growth, aggregation and breakage when the flow field is turbulent. With laminar flow the mixing is not ideal and as a result the steady state well mixed solutions are not accurately simulated.
\end{abstract}

Keywords population balance, particle size distribution, computational fluid dynamics, crystallization, modeling

\section{Introduction}

The population balance equation (PBE) is a statement of continuity for particulate systems. Cases in which a population balance could apply include crystallization, precipitation, bubble columns, gas sparging, sprays, fluidized bed polymerization, granulation, wet milling, liquid-liquid dispersions, air classifiers, hydrocyclones, particle classifiers, and aerosol flows. In the case of a continuous mixedsuspension, mixed-product removal (CMSMPR) crystallizer operating at steady state in which aggregation, breakage and growth are occurring the PBE is given by Randolph and Larson (1988) as

$$
\frac{n(v)-n_{\text {in }}(v)}{\tau}+\frac{\mathrm{d}(G(v) n(v))}{\mathrm{d} v}=b(v)-d(v),
$$

with the boundary condition, $n(0)=n_{0}(x)$. In the above equation $n(v)$ is the number-based population of particles in the tank which is a function of the particle volume, $v$. The subscript "in" refers to the inlet population. $G(v)$ is the volume dependent growth rate and $b(v)$ is the volume dependent birth rate and $d(v)$ is the volume dependent death rate. In the case of aggregation, the birth and death rate terms are given by Hulburt and Katz (1964):

$$
\begin{aligned}
& b_{\mathrm{a}}(v)-d_{\mathrm{a}}(v) \\
& =\int_{0}^{v / 2} \beta(v-w, w) n(v-w) n(w) \mathrm{d} w-n(v) \int_{0}^{\infty} \beta(v, w) n(w) \mathrm{d} w,
\end{aligned}
$$

where the aggregation rate constant, $\beta(v, w)$, is a measure of the frequency of collision of particles of size $v$ with those of size $w$. In the case of breakage, the birth and death rate terms are given by Prasher (1987):

$$
b_{\mathrm{b}}(v)-d_{\mathrm{b}}(v)=\int_{v}^{\infty} S(w) \rho(v, w) n(w) \mathrm{d} w-S(v) n(v),
$$

where $S(v)$ is the breakage rate constant that is a function of particle size, $v, \rho(v, w)$ is the daughter distribution function defined as the probability that a fragment of a particle of size $w$ will appear at size $v$.

It is often useful to know the moments of $n(v)$ because of their physical significance. The $k^{\text {th }}$ volume moment is defined by

$$
{ }_{v} m_{k}=\int_{0}^{\infty} w^{k} n(w) \mathrm{d} w,
$$

where ${ }_{v} m_{0}$ and ${ }_{v} m_{1}$ represent the total number and total volume of particles in the system.

Computational fluid dynamics deals with equations that represent a balance process for mass, momentum, energy and chemical species. These equations are all characterized by the following generalized partial differential equation:

$$
c\left[\frac{\partial \rho \varphi}{\partial t}+\frac{\partial \rho u_{i} \varphi}{\partial x_{i}}\right]=\frac{\partial}{\partial x_{i}} \Gamma^{(\varphi)} \frac{\partial \varphi}{\partial x_{i}}+S^{(\varphi)},
$$

Transient Convective Diffusive Source For the momentum balance, $\varphi$ is given by individual components of the velocity vector, $\Gamma$ is given by the viscosity and there are no source terms. For the energy balance, $\varphi$ is given by temperature; $\Gamma$ is given by the thermal conductivity and the source term given by the heat of reaction or other heat sources. For the mass balance, $\varphi$ is given by mass fraction; $\Gamma$ is given by the molecular diffusion coefficient and the source term given by the rate of chemical reaction.

The population balance equation can also be described in this same form as Eq. (5), when it is written in the moment form of the population balance. In this case, $\varphi$ is 
given by several moments of the population of particles, $\Gamma$ is given by the Brownian diffusivity and the source terms are due to breakage and agglomeration. To well characterize a given particle size distribution several moments are used, typically 3 to 6 . In order to solve those partial differential equations for the momentum, mass, energy and population balance, finite element or finite difference methods are used. Fluent uses a special type of finite difference algorithm called the finite volume method. With the quadrature method of moments (QMOM), the population balance is written as a series of moment equations by multiplying Eq. (1) by $v^{k}$ and integrating with respect to $v$ from zero to infinity. These moment equations are used in place of the PBE to approximate the particle size distribution (see Randolph \& Larson (1988)). QMOM was first proposed by McGraw (1997) and further developed by Marchisio et al. (2003). With the QMOM PBE solver in Fluent 6.2, a small number of moments, $N$, (typically 6 ) are used. Moments are approximated by a quadrature approximation that uses $N / 2$ weights, $W_{i}$, and $N / 2$ sizes, $L_{i}$, as follows:

$$
{ }_{L} m_{k}=\sum_{i=1}^{N / 2} W_{i} L_{i}^{k} .
$$

Upon substitution of these weights and sizes into the $N$ moment equations, we have a series of equations that just equals the number of unknowns, $N$, allowing for the solution of the system of equations that constitutes an approximation of the PBE. From the moments the particle size distribution can be reconstituted using a moment transformation (1). For more details of this numerical method see the Fluent User's Guide - Crystallization Sample Case and Data Files.

The moments used in Fluent's version of QMOM are length based and are different from those described by Eq. (4) which are volume based. There is a correspondence between length based moments and volume based moments, as given in Table 1 , where $K_{a}$ means surface area shape factor, and $K_{v}$ means volume shape factor. Noting this correspondence, any length-based moment calculated by Fluent can be compared with the volume based moment predicted from an analytical solution to the population balance and Eq. (4).

Table 1 Correspondence between length based and volume based moments

\begin{tabular}{lcc}
\hline \multicolumn{1}{c}{ Property } & $\begin{array}{c}\text { Volume based } \\
\text { moment }\end{array}$ & $\begin{array}{l}\text { Length based } \\
\text { moment }\end{array}$ \\
\hline Number of particles & ${ }_{v} m_{0}$ & ${ }^{2} m_{0}$ \\
Surface area of particles & ${ }_{v} m_{2 / 3}$ & $K_{a}{ }^{*}{ }_{2} m_{2}$ \\
Volume of particles & ${ }_{v} m_{1}$ & $K_{v}{ }^{*} L m_{3}$ \\
\hline
\end{tabular}

In this paper a 2-D analogue is used to simulate a stirred tank (CMSMPR) within Fluent. These simulations are compared to steady-state analytical solutions to the PBE for 1) growth, 2) aggregation, 3) breakage, taking place separately and 4) combined nucleation and growth and 5) combined nucleation, growth and aggregation taking place. The analytical solutions for $n(v)$ are converted to the length based moments 0 to 5 and compared directly to the length based moments predicted by Fluent.

\section{Setup of 2-D Stirred Tank in Fluent}

To approximate a well-mixed stirred tank with a simplified computational geometry we have used a 2-D approximation of a stirred tank. This allows testing of the PBE within Fluent simply without solving a complicated 3-D flow problem with rotating grids typical of a stirred tank. The 2-D grid developed is given in Fig. 1.

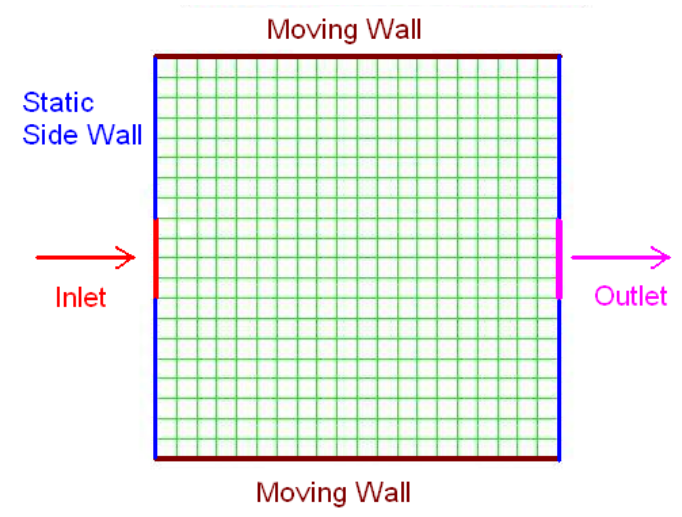

Fig. 1 Grid for 2-D simulation of well mixed stirred tank.

It is a square box $0.1 \mathrm{~m}$ on edge with 400 elements with an inlet and an outlet both with a $0.02 \mathrm{~m}$ opening. The inlet boundary condition is set to inlet velocity of $0.005 \mathrm{~m} \cdot \mathrm{s}^{-1}$. The outlet condition is set to outlet pressure of 0 pascal gauge. For a constant stirred tank, the inlet flow rate is equal to the outlet allowing the mean residence time to be calculated from the inlet flow rate (velocity times inlet area) and the "volume" (box area times unit depth) of the box. To simulate the agitation in the tank the top and bottom walls are assumed to have an $x$-velocity of +101 and $+100 \mathrm{~m} \cdot \mathrm{s}^{-1}$ both in the direction of the outlet for the turbulent case and -8 and $+8 \mathrm{~m} \cdot \mathrm{s}^{-1}$ each in the opposite direction in the laminar case. In the turbulent flow case, $k-\varepsilon$ model is used in addition to the momentum balance for the Reynold's averaged flow field. In the laminar flow case, only the momentum balance is used. The velocity vector field for the laminar case is shown in Fig. 2 and that for the turbulent case is shown in Fig. 3. The convective flux of the tracer at outlet is collected from this simulation and plotted against time (Choi et al., 2004), then converted to residence time distribution (RTD) using:

$$
E(t)=\frac{\mathrm{d} C(t)}{\mathrm{d} t}
$$

The RTD determined in this way is normalized since the feed tracer concentration was 1.0. 


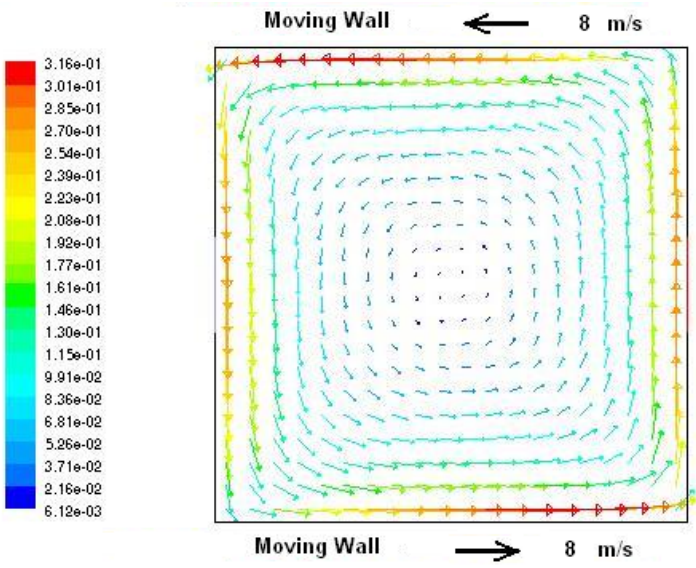

Fig. 2 Velocity distributions for laminar flow Fluent simulation.
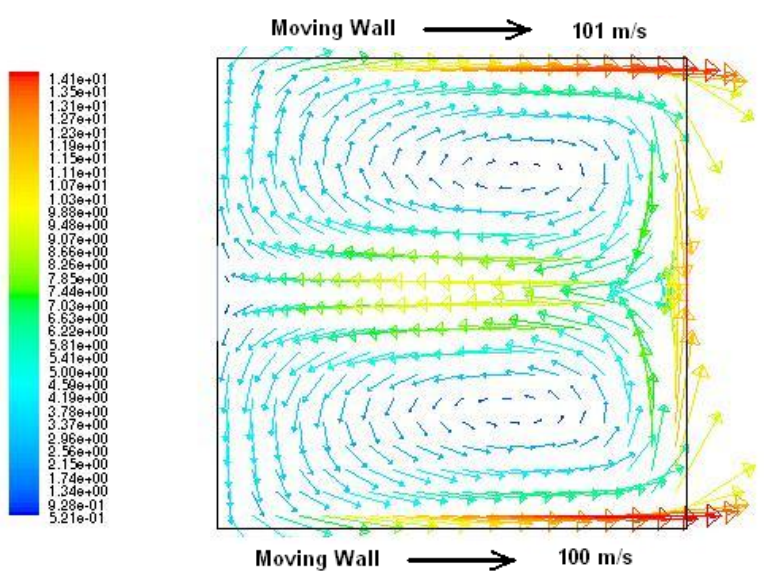

Fig. 3 Velocity distributions for turbulent flow Fluent simulation.

To test the accuracy of the well mixed assumption, the residence time distribution was predicted using a unit tracer concentration, a second phase with the properties of water, in the tank that is allowed to displace a first water phase as time progresses. The tracer is added at the inlet and the concentration of the tracer is monitored at the outlet. The outlet concentration predicted by the simulation is shown in Fig. 4 for the laminar flow and the turbulent flow simulations as well as the ideal curve. Here we see that the laminar flow curve has an initial peak above the ideal curve and a tail that is below the ideal curve, while the turbulent simulation is nearly identical to the ideal curve.

The mean and standard deviation of the various residence time distributions were determined giving the following comparison in Table 2.

Table 2 Comparison of the mean and standard deviation of the residence time distributions

\begin{tabular}{lcccc}
\hline & $\begin{array}{l}t_{\text {mean }} \\
(V / Q)\end{array}$ & Error/\% & Std.Deviation/ $t_{\text {mean }}$ & Error\% \\
\hline Turbulence model & 1.001 & 0.1 & 1.008 & 0.8 \\
Laminar model & 0.999 & 0.1 & 1.058 & 5.8 \\
Perfect mixing & 1 & 0 & 1 & 0 \\
\hline
\end{tabular}

Ideal values for both the mean time, $t_{\text {mean }}$, divided by the ratio of tank volume, $V$, to volumetric flow rate, $Q$ and the standard deviation divided by the mean time should be 1.0 . The laminar flow model is clearly worse than the turbulent flow model in approximating an idealized well-mixed tank.

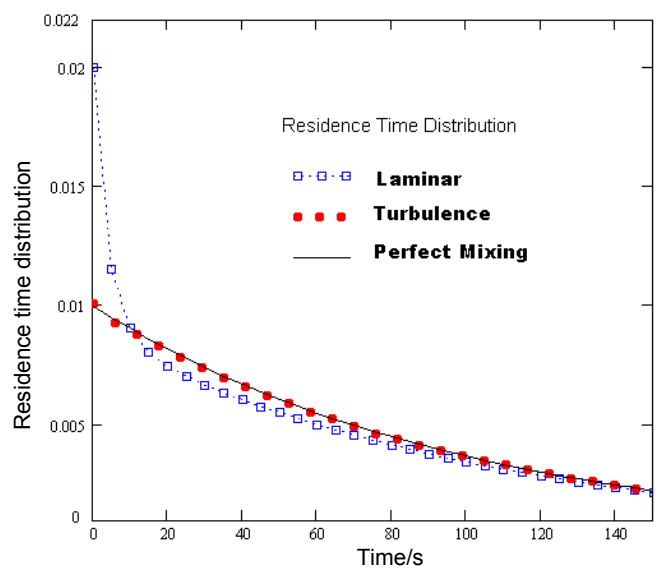

Fig. 4 Comparison of residence time distributions for laminar ( $\square$ ) and turbulent $(\bullet)$ flow simulations with ideal well-mixed tank $(-)$ with the Equation, $E(t)=\exp (-t / \tau), \tau=V / Q$.

\section{Numerical Case Studies}

Numerical cases have been developed to test the PBE capabilities of Fluent. First of all, Fluent is used to solve the velocity field to a convergence of $10^{-5}$ for either the laminar or turbulent flow. Then a particulate multiphase calculation is initiated with the PBE solved by QMOM using 6 lengthbased moments 0 to 5 (or more precisely 3 lengths and 3 weights) with the velocity field fixed. The convergence criterion is lowered to $10^{-7}$ (or lower) for the multiphase PBE calculation with a relaxation parameter of 0.9 except when otherwise stated.

Case 1 - Growth: The analytical solution to the PBE, equation (1), for growth alone was obtained by setting the growth rate to a constant $\left(G(v)=G_{0}\right)$, the aggregation kernel, $\beta(v, w)$, and the specific rate of breakage, $S(v)$, to zero. For this case the feed particle size distribution is

$$
n_{\text {in }}(v)=\frac{N_{0}}{v_{0}} \exp \left(\frac{-v}{v_{0}}\right),
$$

and the boundary condition is set to zero,

$$
n(0)=0 \text {. }
$$

Although not physically realistic, a constant growth rate results in a PBE solution, that is,

$$
n(v)=\exp \left[\frac{-v}{G_{0} \tau}\right]\left[-N_{0} \frac{-1+\exp \left[-v \frac{-v_{0}+G_{0} \tau}{G_{0} \tau v_{0}}\right]}{-v_{0}+G_{0} \tau}\right],
$$

where $\tau$ is the mean residence time. Simulations are performed with $N_{0}$ and $v_{0}$ set equal to unity. Please note that this solution is singular when $v_{0}=\tau G_{0}$. 
Analytical expressions (Nicmanis \& Hounslow, 1998) for the zero ${ }^{\text {th }}$, first and second volume based moments are

$$
\begin{aligned}
& { }_{v} m_{0}={ }_{v} m_{0, \mathrm{in}} \\
& { }_{v} m_{1}={ }_{v} m_{1, \mathrm{in}}+\tau G_{0}{ }_{v} m_{0} . \\
& { }_{v} m_{2}={ }_{v} m_{2, \mathrm{in}}+2 \tau G_{0}{ }_{v} m_{1} .
\end{aligned}
$$

These and other moment equations are used for comparison with Fluent simulations. Because in the analytical solution, the growth term is expressed in terms of the particle volume, $v$, and in Fluent it needs to be expressed in terms of particle length, $x$, we need to perform moment transformations discussed above and presented in Table 1. In addition the growth rate, $G(v)$ used in Eq. (1) and $G_{0}$ used in Eq. (10), is a volume based growth rate that should be converted to a length based growth rate for utilization within Fluent. This transformation is given by

$$
G_{L}(x)=\frac{G(v(x))}{3 K_{v} x^{2}}
$$

where $K_{v}$ is the volume shape factor, a proportionality constant that converts the cube of the size coordinate, $x$, to the particle volume.

For the Fluent simulation the growth rate of $1 \mu \mathrm{m} \cdot \mathrm{s}^{-1}$ and the mean residence time of $100 \mathrm{~s}$ were used with the initial particle size distribution in the tank given by the feed distribution, Eq. (8), the simulation took 1600 iterations to achieve a residue of $10^{-9}$ for the turbulent flow simulation with a relaxation factor of 1.0 and 4000 iterations to achieve a residue of $10^{-9}$ for the laminar flow simulation. The results of these simulations are given in Table 3 for both the laminar and turbulent flow simulations with a relaxation factor of 0.9 .

Table 3 Moment comparison of PBE for Fluent simulations with analytical solution for growth alone

\begin{tabular}{ccccccc}
\hline & & \multicolumn{5}{c}{ Outlet } \\
\cline { 3 - 7 } & \multirow{2}{nnyyyyy}{} & \multirow{2}{*}{ Inlet } & Analytical & \multicolumn{4}{c}{ Fluent } \\
\cline { 3 - 7 } & & & Turbulence & Error/\% & Laminar & Error/\% \\
\hline${ }_{L} m_{0}$ & 1 & 1 & 0 & 1 & 0 \\
${ }_{L} m_{1}$ & 1.108 & 5.183 & 5.1906333 & 0.147 & 5.0913844 & 1.768 \\
${ }_{L} m_{2}$ & 1.39 & 30.227 & 30.251846 & 0.082 & 29.813738 & 1.367 \\
${ }_{L} m_{3}$ & 1.91 & 192.896 & 193.02859 & 0.069 & 192.86899 & 0.014 \\
${ }_{L} m_{4}$ & 2.821 & 1323.3 & 1325.7223 & 0.206 & 1347.62 & 1.861 \\
${ }_{L} m_{5}$ & 4.423 & 9626.3 & 9641.8662 & 0.165 & 9985.3242 & 3.733 \\
\hline
\end{tabular}

The turbulent flow case will not converge with a relaxation factor of 1.0. The results of the turbulent flow simulation are accurate to only $0.2 \%$ with this convergence criterion. The results of the laminar flow simulation is less accurate - a 3.7\% error with the same convergence criterion. Because the laminar flow simulation does not correspond to well-mixed conditions, and therefore does not accurately simulate the analytical solution. This is shown in Fig. 5 and Fig. 6, in which a contour plot of ${ }_{L} m_{1}$ for turbulent conditions and laminar conditions are shown respectively. We can see that with turbulent conditions ${ }_{L} m_{1}$ is nearly constant everywhere inside tank except near the inlet where $\left\llcorner m_{1}\right.$ is near zero, while for laminar flow conditions, $L m_{1}$ is not evenly distributed. In the center of the tank, the value of ${ }_{L} m_{1}$ is larger than that near the walls. In the center of the flow field, the fluid stays longer than near the walls due to a dead zone (see Fig. 2) thus leading to a longer growth period to give larger particles and therefore larger values of ${ }_{\llcorner} m_{1}$.
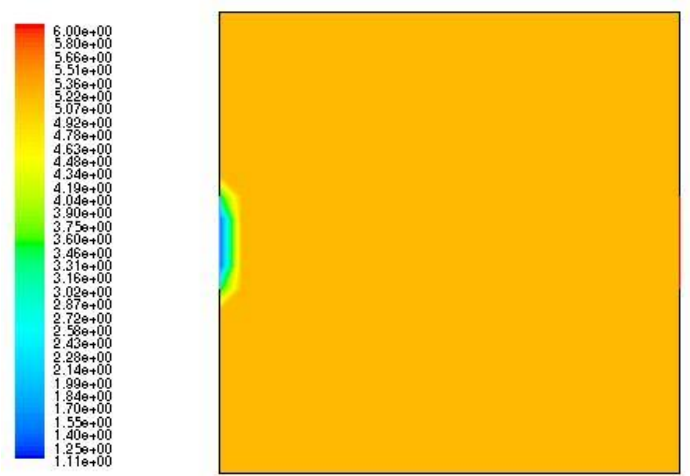

Fig. 5 Contour plot of ${ }_{L} m_{1}$ for 2-d tank operated with turbulent flow conditions.

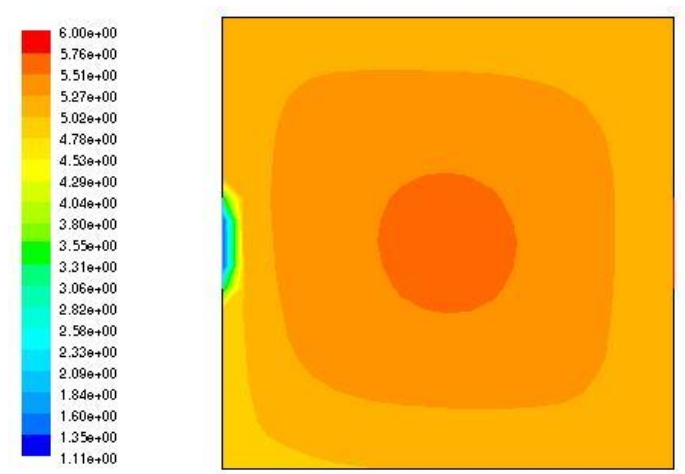

Fig. 6 Contour plot of ${ }_{L} m_{1}$ for 2 -d tank operated with laminar flow conditions.

Case 2 - Nucleation and growth: The analytical solution to the PBE, Eq. (1), for nucleation and growth, was obtained by setting the growth rate to a constant $\left(G(v)=G_{0}\right)$, the aggregation kernel, $\beta(v, w)$, and the specific rate of breakage, $S(v)$, to zero. For this case the feed particle size distribution is set to zero, $n_{\text {in }}(v)=0$ and the boundary condition is

$$
n(0)=n_{0}
$$

where $n_{0}$ is the number density of particles with a zero size. The nucleation rate is given by the product of $G_{0}$ and $n_{0}$. The analytical solution for this case is given by Randolph and Larson (1988):

$$
n(x)=n_{0} \exp \left(\frac{-x}{G_{0} \tau}\right)
$$

This analytical solution is converted to length-based moments for comparison with the Fluent simulation. The 
Fluent simulation was run with $G_{L-0}=0.01 \mathrm{~mm} \cdot \mathrm{s}^{-1}$, noting the above conversion in Eq. (12) and the nucleation rate of $1 \mathrm{~m}^{-3} \cdot \mathrm{s}^{-1}$, and the mean residence time $\tau$ of $100 \mathrm{~s}$ with no particles in the feed. The results of this comparison are given in Table 4. Here we see that the laminar flow simulation is in error by as much as $\sim 25 \%$ while the turbulent flow simulation is accurate to $\sim 0.01 \%$.

Table 4 Moment comparison of PBE for Fluent simulations with analytical solution for nucleation and growth

\begin{tabular}{ccccccc}
\hline & & \multicolumn{5}{c}{ Outlet } \\
\cline { 3 - 7 } & \multirow{2}{*}{ Inlet } & \multirow{2}{*}{ Analytical } & \multicolumn{5}{c}{ Fluent } \\
\cline { 3 - 7 } & & & Turbulence & Error/\% & Laminar & Error/\% \\
\hline${ }_{L} m_{0}$ & 0 & 100 & 99.987 & 0.013 & 99.98 & 0.02 \\
${ }_{L} m_{1}$ & 0 & 100 & 99.988 & 0.012 & 105.68 & 5.68 \\
${ }_{L} m_{2}$ & 0 & 200 & 199.977 & 0.012 & 223.2 & 11.6 \\
${ }_{L} m_{3}$ & 0 & 600 & 599.933 & 0.012 & 702.3 & 17.5 \\
${ }_{L} m_{4}$ & 0 & 2400 & 2399.732 & 0.011 & 2917.2 & 21.55 \\
${ }_{L} m_{5}$ & 0 & 12000 & 11998.66 & 0.011 & 14982 & 24.85 \\
\hline
\end{tabular}

Case 3 - Aggregation: The analytical solution to the PBE, Eq. (1), for aggregation alone was obtained by setting the growth rate to zero $(G(v)=0)$, the aggregation kernel to a constant, $\beta(v, w)=\beta_{0}$, and the specific rate of breakage, $S(v)$, to zero. For this case the feed particle size distribution is set to an exponential distribution given by Eq. (8).

The analytical solution for this case is given by Hounslow (1990):

$$
n(v)=\frac{N_{0}}{v_{0}} \frac{I_{0}\left[\frac{-\left(\beta_{0} N_{0} \tau\right) v}{v_{0}\left[1+2\left(\beta_{0} N_{0} \tau\right)\right]}\right]+I_{1}\left[\frac{-\left(\beta_{0} N_{0} \tau\right) v}{v_{0}\left[1+2\left(\beta_{0} N_{0} \tau\right)\right]}\right]}{\sqrt{1+2\left(\beta_{0} N_{0} \tau\right)} \exp \left[\frac{\left(1+\beta_{0} N_{0} \tau\right) v}{\left[1+2\left(\beta_{0} N_{0} \tau\right)\right] v_{0}}\right]}
$$

where $I_{0}(z)$ and $I_{1}(z)$ are modified Bessel Functions of the first kind of zero and first orders. This analytical solution is converted to length-based moments for comparison with the Fluent simulation. Analytical expressions (Smit et al., 1993) for the zero ${ }^{\text {th }}$, first and second volume based moments are

$$
\begin{aligned}
& { }_{v} m_{0}=\frac{-1+\sqrt{1+2 \beta_{0} m_{0, \mathrm{in}} \tau}}{\beta_{0} \tau} \\
& { }_{v} m_{1}={ }_{v} m_{1, \mathrm{in}} \\
& { }_{v} m_{2}={ }_{v} m_{2, \text { in }}+\tau \beta_{0}{ }_{v} m_{1}^{2}
\end{aligned}
$$

The Fluent simulation was run for the conditions of $\beta_{0}=1 \mathrm{~m}^{4} \cdot \mathrm{s}^{-1}, N_{0}=100 \mathrm{~m}^{-3}, v_{0}=100 \mu \mathrm{m}$, and a mean residence time of $100 \mathrm{~s}$ with the relaxation factor set to 0.9 . The turbulent simulation ran for 1000 iterations to get to a residue of $10^{-9}$ while the laminar simulation ran for 3000 iterations to get a residue of $10^{-10}$. The results of this comparison are given in Table 5 . Here we see that the turbulent flow simulation is accurate to $\sim 0.4 \%$ and the $3^{\text {rd }}$ length based moment is correctly predicted to not change during passage through the reactor.
Table 5 Moment comparison of PBE for Fluent simulations with analytical solution for aggregation alone

\begin{tabular}{lcccccc}
\hline & & \multicolumn{5}{c}{ Outlet } \\
\cline { 3 - 7 } & \multirow{2}{*}{ Inlet } & \multirow{2}{*}{ Analytical } & \multicolumn{5}{c}{ Fluent } \\
\cline { 3 - 7 } & & & Turbulence & Error/\% & Laminar & Error/\% \\
\hline${ }_{L} m_{0}$ & 1 & 0.132 & 0.1319 & 0.076 & 0.1593889 & 20.75 \\
${ }_{L} m_{1}$ & 1.108 & 0.225 & 0.2256 & 0.267 & 0.2549767 & 13.32 \\
${ }_{L} m_{2}$ & 1.39 & 0.547 & 0.5490 & 0.366 & 0.5764962 & 5.392 \\
${ }_{L} m_{3}$ & 1.91 & 1.91 & 1.91 & 0 & 1.91 & 0 \\
${ }_{L} m_{4}$ & 2.821 & 9.073 & 9.093 & 0.22 & 8.9678564 & 1.159 \\
${ }_{L} m_{5}$ & 4.423 & 53.797 & 53.88 & 0.154 & 53.04977 & 1.389 \\
\hline
\end{tabular}

Case 4 - Breakage: The analytical solution to the PBE, Eq. (1), for breakage alone was obtained by setting the growth rate to zero $(G(v)=0)$, the aggregation kernel to zero, $\beta(v, w)=0$, the specific rate of breakage to $S(v)=v \mathrm{~s}^{-1}$ and the daughter distribution function to $\rho(v, w)=2 / w$. For this case the feed particle size distribution is set to an exponential distribution given by Eq. (8).

The analytical solution for the case is given by Nicmanis and Hounslow (1998):

$$
n(v)=\frac{N_{0}\left[(1+\tau v)^{2}+2 \tau v_{0}\left(1+\tau\left(v_{0}+v\right)\right)\right]}{v_{0}(1+\tau v) \exp \left(\frac{v}{v_{0}}\right)} .
$$

This analytical solution is converted to length-based moments for comparison with the Fluent simulation.

Analytical expressions of the zero ${ }^{\text {th }}$ and first volume moments can be derived to give

$$
\begin{aligned}
& { }_{v} m_{0}=\tau_{v} m_{1}+{ }_{v} m_{0, \text { in }}, \\
& { }_{v} m_{1}={ }_{v} m_{1, \text { in }}
\end{aligned}
$$

which indicate that the volume of particles is conserved. These moments are converted to length-based moments for direct comparison with the QMOM Fluent simulation.

The Fluent simulation was run with constants $N_{0}$ and $v_{0}$ set to unity and the mean residence time $\tau$ to $100 \mathrm{~s}$. Comparison of the Fluent simulations to the analytical solution for both the laminar and turbulent flow simulations is given in Table 6.

Table 6 Moment comparison of Fluent simulations with analytical solution for breakage only

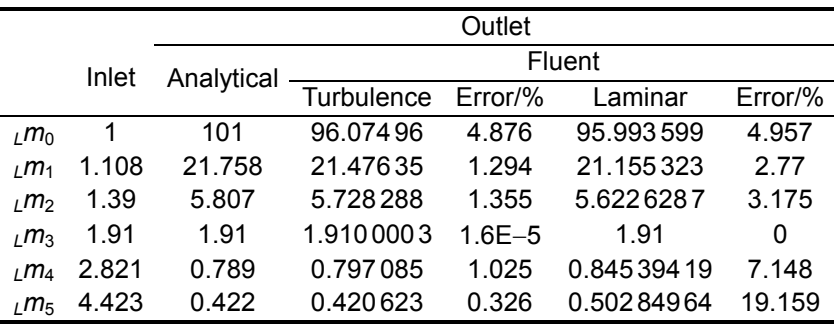

To get to a residue of $10^{-10}$ required 22000 iterations using a relaxation factor of 1.0 for turbulent flow and 9000 iterations with a relaxation factor of 0.9 for the laminar flow simulation. The results for ${ }_{L} m_{3}$ are accurately predicted indicating that mass is conserved, and the errors for other moments are within $4.9 \%$. This indicates that Fluent QMOM does accurately simulate breakage. 
Case 5 - Nucleation, growth and aggregation combined: The analytical solution to the PBE, Eq. (1), for nucleation, growth and aggregation together was obtained by setting the growth rate to a constant $\left(G(v)=G_{0}\right)$, the aggregation kernel to a constant, $\beta(v, w)=\beta_{0}$, and the specific rate of breakage, $S(v)$, to zero. For this case the feed particle size distribution is set to zero, $n_{\text {in }}(v)=0$, and the boundary condition is

$$
n(0)=n_{0}
$$

where $n_{0}$ is the number density of particles with zero size. The nucleation rate is given by the product of $G_{0}$ and $n_{0}$. The analytical solution for this case is given by Liao and Hulburt (1976):

$$
\begin{aligned}
& n(v)= \\
& 2 n_{0} \frac{\exp \left[-\sqrt{1+\frac{1}{2 \beta_{0} n_{0} G_{0} \tau^{2}}}\left(\frac{v}{G_{0}} \sqrt{2 \beta_{0} n_{0} G_{0}}\right)\right]}{\frac{v}{G_{0}} \sqrt{2 \beta_{0} n_{0} G_{0}}} I_{1}\left(\frac{v}{G_{0}} \sqrt{2 \beta_{0} n_{0} G_{0}}\right),
\end{aligned}
$$

where $I_{1}(z)$ is the modified Bessel Function of the first kind of first order. Analytical expressions of the zeroth, first and second volume moments can be derived to give

$$
\begin{aligned}
{ }_{v} m_{0} & =\frac{-1+\sqrt{1+2 \beta_{0} n_{0} G_{0} \tau}}{\beta_{0} \tau} \\
{ }_{v} m_{1} & =\tau G_{0} m_{0} \\
{ }_{v} m_{2} & =2 \tau G_{0} m_{1}+\tau \beta_{0}{ }_{v} m_{1}^{2}
\end{aligned}
$$

This analytical solution and the above moment equations are converted to length based moments for comparison with the Fluent simulation. The Fluent simulation was run with $G_{v-0}=0.01 \mathrm{~mm}^{3} \cdot \mathrm{s}^{-1}$, the aggregation kernel $\beta_{0}=0.1 \mathrm{~m}^{4} \cdot \mathrm{s}^{-1}$ and the nucleation rate of $0.01 \mathrm{~m}^{-3} \cdot \mathrm{s}^{-1}$, the mean residence time $\tau$ of $100 \mathrm{~s}$ and with no particles in the feed. The solution took 8000 iterations to reach a residue of $10^{-10}$ for the turbulent simulation and 7000 iterations to reach a residue of $10^{-9}$ for the laminar flow simulation. To get convergence with the laminar flow simulation the relaxation factor for the population balance was initially set for 0.5 and after 1000 iterations it was raised to 0.8 . The results of this comparison are given in Table 7 . For the turbulent simulation, the largest error is $1.4 \%$ in the length moment, ${ }_{L} m_{1}$ and for the laminar flow simulation, the largest error is $7.539 \%$ for the ${ }_{L} m_{5}$ moment.

Table 7 Moment comparison of FLUENT simulation with analytical solution to the PBE for nucleation, growth and aggregation

\begin{tabular}{ccccccc}
\hline & & \multicolumn{5}{c}{ Outlet } \\
\cline { 3 - 7 } & Inlet & Analytical & \multicolumn{5}{c}{ Fluent } \\
\cline { 3 - 7 } & & & Turbulence & Error/\% & Laminar & Error/\% \\
\hline${ }_{L} m_{0}$ & 0 & 0.358 & 0.3582 & 0.056 & 0.3443651 & 3.809 \\
${ }_{L} m_{1}$ & 0 & 0.346 & 0.3508 & 1.387 & 0.3394129 & 1.904 \\
${ }_{L} m_{2}$ & 0 & 0.434 & 0.4367 & 0.622 & 0.4296504 & 1.002 \\
${ }_{L} m_{3}$ & 0 & 0.684 & 0.6845 & 0.073 & 0.6909166 & 1.011 \\
${ }_{L} m_{4}$ & 0 & 1.305 & 1.3178 & 0.981 & 1.3717375 & 5.114 \\
${ }_{L} m_{5}$ & 0 & 2.904 & 2.9091 & 0.176 & 3.1229196 & 7.539 \\
\hline
\end{tabular}

\section{Conclusions}

A 2-D model of a well-mixed stirred tank using a simple geometry with a small number of grids can be shown to be an accurate model of a well-mixed crystallizer if the flow is turbulent. Using this turbulent model of a well-mixed tank, a two-phase model with a PBE for the second, solid phase has been developed and solved with the QMOM option within Fluent 6.2. This model has been tested using numerical cases where growth, aggregation, breakage and the combined cases of nucleation and growth and nucleation, growth and aggregation. These Fluent simulations are compared with analytical solutions to the PBE for a constantly well-mixed tank for these cases. The QMOM option in Fluent accurately predicts each of these cases. To obtain less than $1 \%$ accuracy for these cases, different convergence criteria are necessary. Depending upon the case, a convergence criterion between $10^{-7}$ to $10^{-14}$ is required. A laminar model with poorer mixing conditions is used for comparison purposes. The results of the laminar model are far away from the analytical solutions, indicating that mixing conditions are very important for crystallizer performance; different mixing condition will results in different product particle size distributions.

\section{References}

Choi, B.-S., Wan, B., Philyaw, S., Dhanasekharan, K. \& Ring, T. A. (2004). Residence time distributions in a stirred tank: comparison of CFD predictions with experiment. Ind. Eng. Chem. Res., 43(20), 6548-6556

Hounslow, M. J. (1990). A discrete population balance for continuous systems at steady state. AIChE J., 36(1), 106.

Hulburt, H. M. \& Katz, S. (1964). Some problems in particle technology - statistical mechanical formulation. Chem. Eng. Sci., 19, 555 .

Liao, P. F. \& Hulburt, H. M. (1976). Agglomeration processes in suspension crystallization. AlChE Meeting, Chicago, December, 1976.

Marchisio, D. L., Virgil, R. D. \& Fox, R. O. (2003). Quadrature method of moments for aggregation-breakage processes. J. Colloid Interface Sci., 258, 322-334.

McGraw, R. (1997). Description of aerosol dynamics by quadrature method of moments. Aerosol Sci.Technol., 27, 255-265.

Nicmanis, M. \& Hounslow, M. J. (1998). Finite-element methods for steady-state population balance equations. AIChE J., 44(10), 2258-2272.

Prasher, C. L. (1987). Crushing and Grinding Process Handbook. New York: Wiley.

Randolph, A. D. \& Larson, M. A. (1988). Theory of Particulate Processes $\left(2^{\text {nd }}\right.$ ed. $)$. New York: Academic Press.

Smit, D. J., Hounslow, M. J. \& Paterson, W. R. (1993). Aggregation and gelation 1: analytical solutions for CST and batch operation. Chem. Eng. Sci, 49(7), 1025.

Manuscript received February 15, 2005 and accepted July 12, 2005. 